\section{Alternativa para el tratamiento de la atrofia severa de maxilar superior: casos clínicos de implantes cigomáticos}

\section{Zygomatic implants for the management of severe alveolar atrophy: clinical cases}

\section{Resumen}

La rehabilitación implanto-protética de maxilares con atrofia severa, presenta un desafío en la odontología actual. La falta de tejido óseo para la colocación de implantes estándares, conlleva tener que decidir una alternativa de tratamiento para el paciente. El objetivo de esta publicación es presentar dos casos clínicos del tratamiento de la atrofia de maxilar superior a través de implantes cigomáticos. Se presentan dos casos clínicos operados mediante la metodología descrita por Branemark, debatiendo las alternativas quirúrgicas actuales. Una paciente presentó dolor peri-orbitario, que fue resuelto inmediatamente. No se presentaron otras complicaciones post quirúrgicas. Se discuten alternativas protéticas de la resolución de estos casos. Transcurridos 12 y 18 meses de la rehabilitación implanto protética, no se presentaron otras complicaciones quirúrgicas ni protéticas en ambos pacientes. Se concluye que la rehabilitación con implantes cigomáticos es una alternativa válida para rehabilitar los maxilares superiores atróficos, de corto tiempo, baja morbilidad, y que presenta tasas de éxito similares a los implantes convencionales.

Palabras clave: Atrofia; Arcada edéntula; Maxilar superior; Implantes dentales; Cigoma (fuente: DeCS BIREME).

\begin{abstract}
Implant-prosthetic rehabilitation of severe atrophic maxilla is a challenge for clinicians. Decision making when patients lack of bone density or quantity is usually difficult. The aim of this study is to report two cases of severe atrophic maxilla treated through zygomatic implants. Two clinical reports using the Branemark methodology are presented, in which surgical alternatives are discussed. One patient had post-surgical pain in the peri orbital area and could be solved immediately. No other surgical complications were observed. Prosthetic alternatives are also discussed. After 12 and 18 months of functional loading, neither surgical nor prosthetic problems occurred. In conclusion, zygomatic implants are a viable, short time, low morbidity treatment in patients with an atrophic maxilla and present success rates similar to conventional implants.
\end{abstract}

Keywords: Atrophy; Edentulous jaw; Maxilla; Dental implants; Zygoma (source: MeSH NLM).

\section{Caso Clínico}

Carlos Lazarte ${ }^{1, a}$, Carlos Farias-Scroppo ${ }^{1, b}$, Gonzalo Oviedo ${ }^{1, c}$, Matias Garcia-Blanco ${ }^{1, d}$, Sebastián Ariel Puia ${ }^{1, d}$

${ }^{1}$ Universidad de Buenos Aires, Facultad de Odontología, Cátedra de Cirugía y Traumatología Buco-Máxilo-Facial I, Buenos Aires, Argentina.

${ }^{a}$ Médico Cirujano.

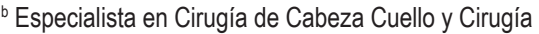
Máxilo-Facial.

${ }^{c}$ Cirujano Dentista.

d Doctor en Odontología.

\section{Correspondencia:}

Matias Garcia-Blanco.

Correo electrónico: matiasgarciablanco@yahoo.com.ar Av. De Los Incas 3295 5to37, Buenos Aires, Argentina. CP 1426.

ORCID: 0000-0003-3974-8756

\author{
Coautores: \\ Carlos Lazarte \\ carloslazarte75@hotmail.com \\ Carlos Farias-Scroppo \\ cfariasscroppo@gmail.com \\ Gonzalo Oviedo \\ gjoviedo@gmail.com \\ Sebastián Ariel Puia \\ sebapuia@hotmail.com
}

\section{Editora:}

Sandra Patricia Palomino-Gómez

Universidad Nacional Mayor de San Marcos, Perú.

Conflictos de intereses: sin conflicto de intereses.

Fuente de financiamiento: autofinanciamiento.

\section{Recibido: 26/08/19 \\ Aceptado: 30/11/19}

Publicado: 24/02/20 


\section{Introducción}

La pérdida de las piezas dentarias se asocia con una serie de procesos fisiológicos que determinan la reabsorción progresiva del reborde alveolar; que en estadios avanzados, puede conllevar la perdida acentuada del capital óseo. La reposición de estructuras dentarias mediante implantes dentales en la atrofia severa de maxilar superior representa un desafío ${ }^{1}$. Para solucionar esta deficiencia de capital óseo, se han sugerido varios procedimientos de regeneración ósea, como la elevación del piso del seno maxilar, la regeneración tisular guiada, la distracción osteogénica, y los injertos en bloque ${ }^{2,3}$.

Las mencionadas técnicas necesitan tiempo para la estabilización e integración del injerto con el hueso nativo del paciente, alargando los tiempos de tratamiento. Además, presentan una serie de morbilidades inherentes, presentes en todas las cirugías de regeneración ósea como el dolor, la inflamación, y el edema; y la posibilidad de dehiscencia de la herida, o infección del injerto. Incluso superada la etapa de cicatrización inicial, es de esperar una reabsorción variada del injerto óseo colocado. Alternativamente a las técnicas de regeneración ósea, se han propuesto: la colocación de implantes cortos, la utilización de implantes inclinados, y los implantes cigomáticos ${ }^{4,5}$.

En 1993, Aparicio y cols. ${ }^{6}$ mencionaron la posibilidad de colocar implantes dentales en la región cigomática; y en 1997 , Weischer y cols. ${ }^{7}$ describieron el uso del cigoma como estructura de anclaje primario en la rehabilitación de pacientes sometidos a maxilectomías. También Branemark, en 1998, presentó un protocolo quirúrgico considerado la técnica convencional, que posteriormente algunos autores agregaron variantes ${ }^{8}$. Esta técnica consiste en la realización de una ostectomía alta en la pared anterior del seno maxilar para lograr la visualización del antro durante la secuencia de fresado, y colocación del implante cigomático.

El hueso malar utilizado para anclar los implantes cigomáticos se caracteriza por un hueso cortical con una estructura trabecular densa, muy favorable para el proceso de oseointegración ${ }^{9}$. Algunos estudios han evaluado la colocación de cuatro implantes cigomáticos distribuidos en dos, en ambos lados de la línea media, sin soporte de implantes estándares en la pre-maxila, obteniendo tasas de sobrevida implantaria similar a los implantes estándares ${ }^{10,11}$.

Las principales ventajas de la técnica de los implantes cigomáticos radica en que no requieren de regeneración ósea, acortando considerablemente los tiempos de tratamiento, y reduciendo la cantidad de intervenciones quirúrgicas necesarias. Se ha destacado que cuando se utiliza esta modalidad de tratamiento, los pacientes han demostrado satisfacción con sus condiciones masticatorias, fonéticas, estéticas y psicológicas ${ }^{10}$.

El objetivo de esta publicación es presentar dos casos clínicos de una alternativa de tratamiento para maxilares superiores atróficos, a través de los implantes cigomáticos.

\section{Reporte de los casos}

\section{Caso clínico 1}

Paciente de sexo femenino de 57 años de edad, sin patología sistémica asociada, ni hábitos parafuncionales, ni consumo de alcohol y tabaco, concurrió al servicio solicitando una rehabilitación odontológica. Se realizó la anamnesis, la evaluación clínica y por imágenes (Figura 1), y el montaje de modelos de estudio. La paciente presentaba edentulismo total en ambos maxilares habiendo perdido las piezas dentarias por periodontitis generalizada desde la adolescencia. Presentaba una atrofia severa de maxilar superior con reabsorción alveolar basal inicial, clase D en la clasificación de Lekholm y Zarb. Se evaluaron opciones de regeneración ósea, y alternativamente la colocación de cuatro implantes cigomáticos sin regeneración ósea. Evaluando las alternativas con la paciente, de decidió realización de implantes cigomáticos para rehabilitar el maxilar superior, para disminuir la cantidad de intervenciones quirúrgicas y para acortar el tiempo de tratamiento. Se solicitó a la paciente firmar el consentimiento de la cirugía, y para la utilización de las imágenes. La paciente fue tratada quirúrgicamente con anestesia general. Se realizó una incisión contínua palatinizada del reborde alveolar y dos descargas verticales

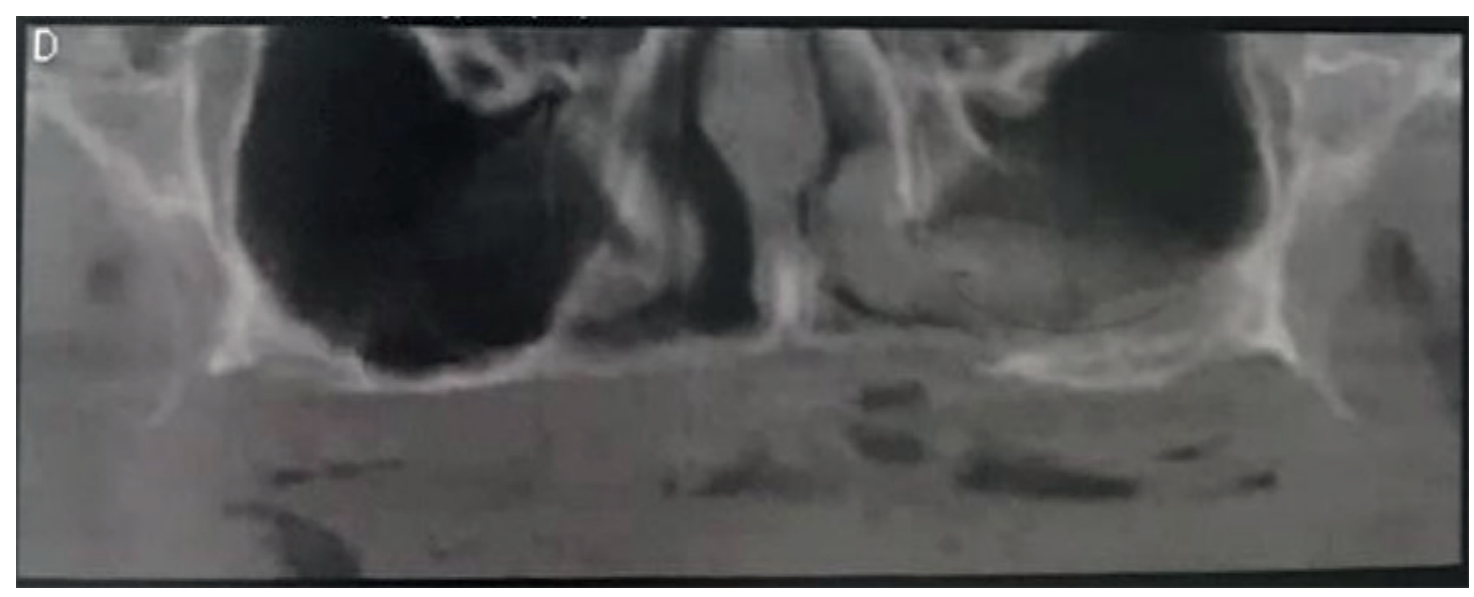

Figura 1. Corte paronex de la tomografía volumétrica pre quirúrgica (caso clínico 1) 
distales. Se levantó un colgajo mucoperióstico para exponer el reborde alveolar, la pared lateral del seno maxilar y el borde inferior del arco cigomático. Se realizó una antrotomía simple alta para acceder a la membrana de Schneider, la cual fue legrada cuidadosamente en sus paredes lateral y caudal. Se realizó el paso de tres fresas del sistema implantario, utilizando la técnica intrasinusal descrita por Branemark (TIB) ${ }^{8}$. Se colocaron dos implantes cigomáticos a cada lado de la línea media (de $4 \mathrm{x}$ $40 \mathrm{~mm}$, Neodent, Brasil) (Figura 2). Se prosiguió con la reposición del colgajo y la síntesis de la herida con hilo de seda. Adicionalmente en el maxilar inferior se realizó un injerto de cresta ilíaca para la rehabilitación integral de ambos maxilares. A la paciente se le administraron endovenosamente antibióticos, corticoides, y analgésicos; y posteriormente, vía oral, antibióticos y analgésicos. Se realizó una radiografía panorámica de control (Figura 3). El postoperatorio cursó sin complicaciones, y a los 10 días se retiraron las suturas. A los cuatro meses se realizó el destape de los implantes cigomáticos, observándose una estabilidad clínica de los mismos. Se tomaron impresiones con hidrocoloide irreversible para confeccionar modelos y cubetas individuales. Dos semanas después se colocaron transferencias en los implantes cigomáticos, comprobándose su asentamiento a través de radiografías periapicales. Se ferulizaron las transferencias de cada sector realizando una unión con hilo dental, posteriormente fijada con resina acrílica (Duralay). Se realizó a impresión del maxilar superior con silicona de adición, realizando un remarginado de los bordes externos. Se realizó el montaje de ambos modelos definitivos en un articulador semiajustable, y se evaluó la disposición de los dientes clínicamente según los requisitos estéticos y funcionales antes de avanzar con la prótesis. Se solicitó al laboratorio dental la elaboración de dos barras con sistemas retentivos (Figura 4), y una prótesis sobredentadura teniendo los parámetros dentarios previamente establecidos (Figura 5). Después de la carga oclusal, el paciente informó una mejoría en función y estética (Figura 6). Transcurridos 18 meses de la carga protética, no se han observado problemas implantarios ni protéticos. En el maxilar inferior se realizó posteriormente una prótesis híbrida convencional de mediana complejidad.

\section{Caso clínico 2}

Paciente de sexo femenino de 53 años de edad, con hipotiroidismo controlado, sin otra patología sistémica, sin hábitos parafuncionales, ni consumo de alcohol y tabaco, concurrió al servicio solicitando una rehabilitación odontológica integral. Se realizó la anamnesis, la evaluación clínica y por imágenes (Figura 7), y el montaje de modelos de estudio. La paciente presentaba el maxilar superior edéntulo, y el maxilar inferior parcialmente desdentado, con una historia de pérdida de piezas dentarias por caries dentales incorrectamente tratadas. Presentaba una atrofia severa de maxilar superior con reabsorción alveolar basal avanzada, clase E en la clasificación de Lekholm y Zarb. Se realizaron los estudios pre-quirúrgicos pertinentes, y se decidió la rehabilitación del maxilar superior atrófico a través de implantes cigomáticos, y la instalación de una prótesis parcial removible en el maxilar inferior. Se solicitó a la paciente firmar el consentimiento de la cirugía y de la utilización de imágenes. La paciente fue tratada quirúrgicamente con anestesia general. Se realizó una incisión continua palatinizada del reborde alveolar y dos descargas verticales distales. Se levantó un colgajo mucoperióstico, se realizó una antrotomía simple alta y se legró cuidadosamente la membrana sinusal. Se realizó el fresado quirúrgico, utilizando la TIB. Se colocaron dos implantes cigomáticos a cada lado de la línea media (de 4 × $40 \mathrm{~mm}$,

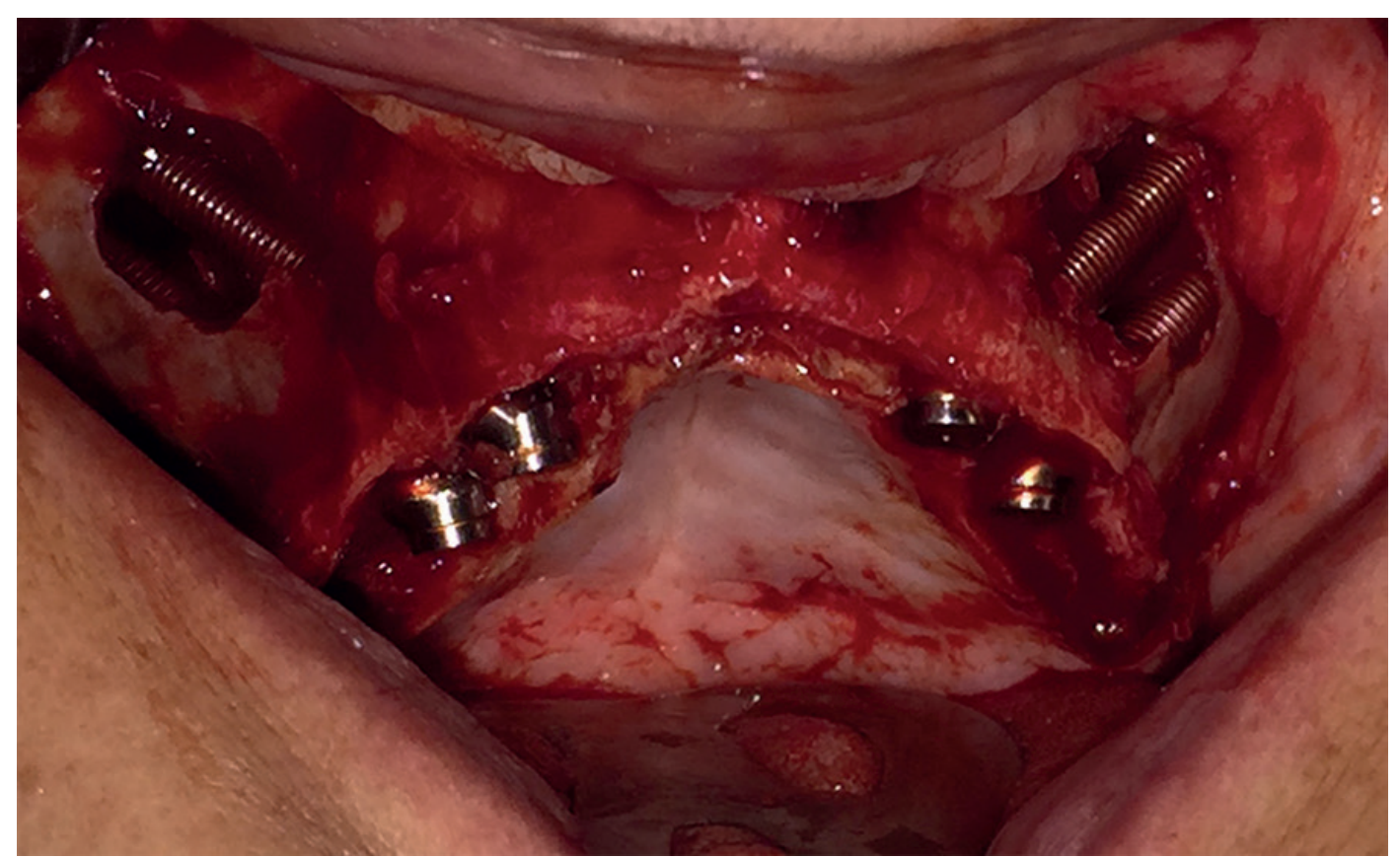

Figura 2. Colocación de cuatro implantes cigomáticos a través de la técnica convencional (caso clínico 1) 


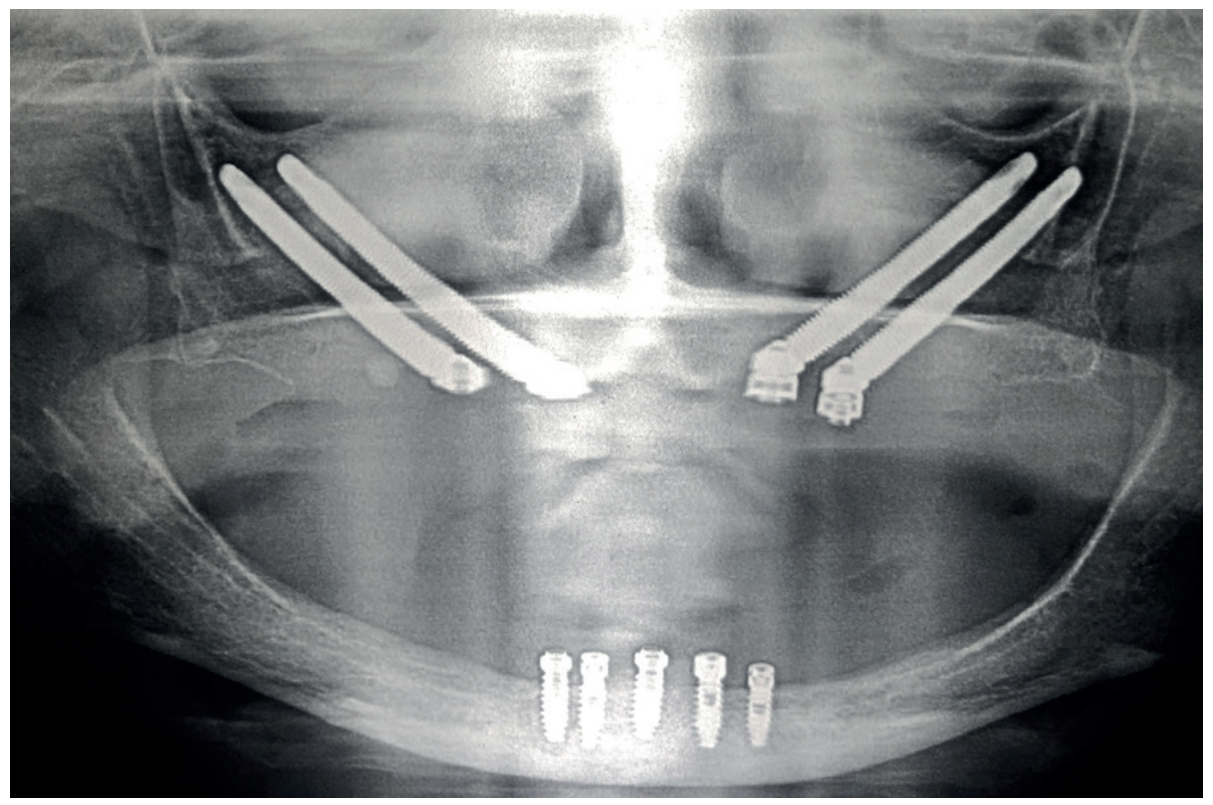

Figura 3. Radiografía panorámica post quirúrgica (caso clínico 1)

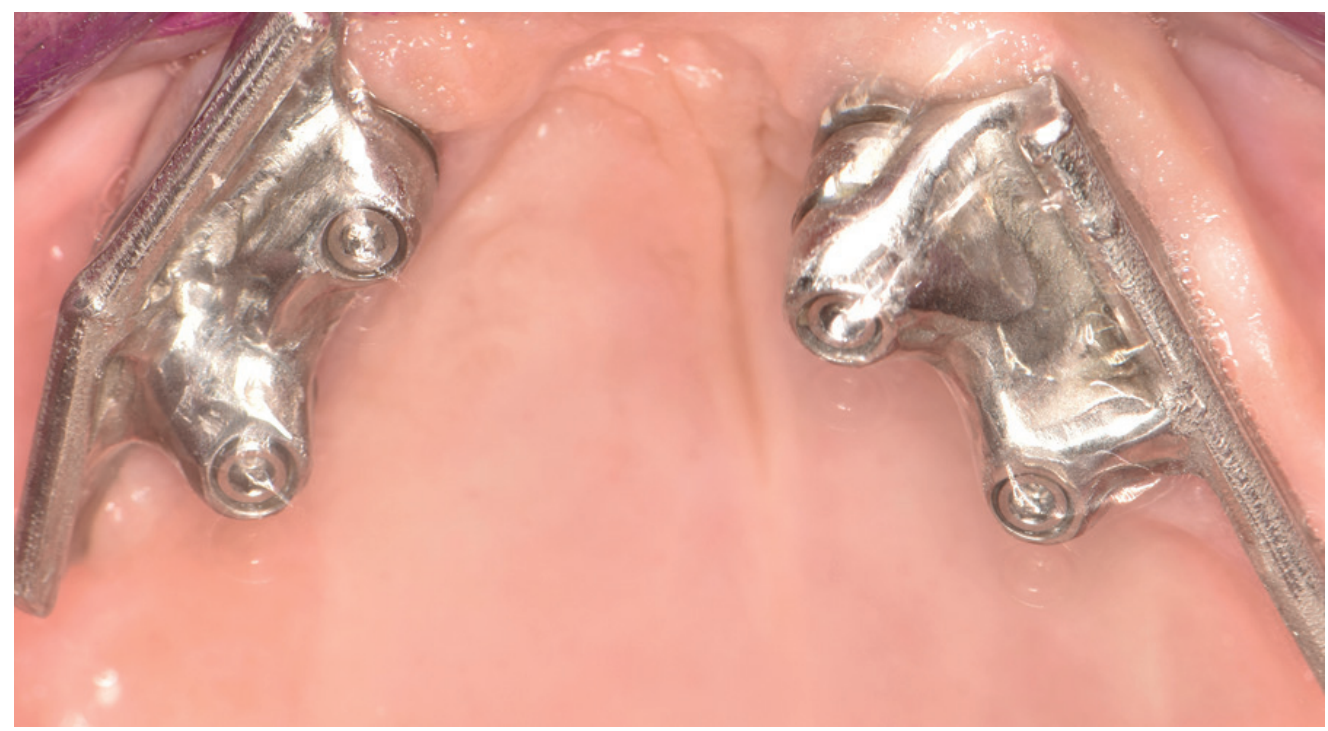

Figura 4. Instalación de dos barras para la confección de prótesis sobredentadura (caso clínico 1)

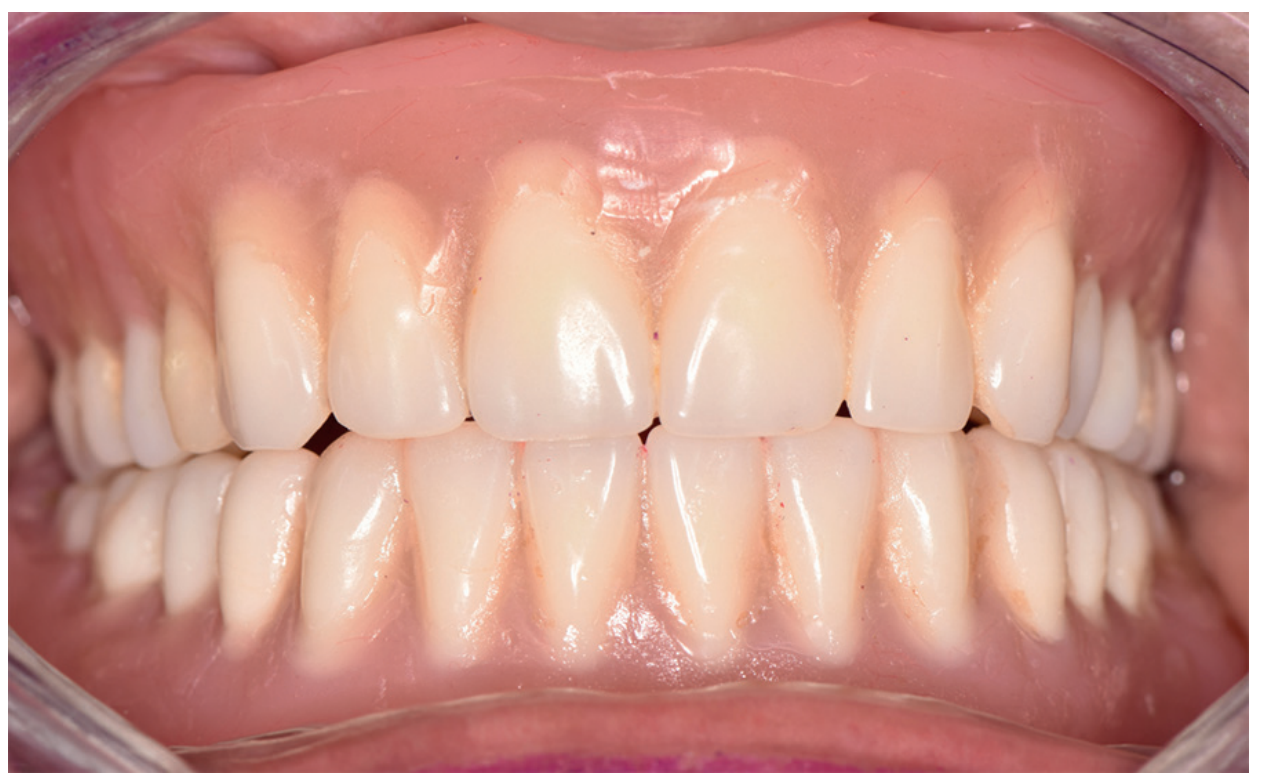

Figura 5. Rehabilitación final del maxilar superior a través de prótesis sobredentadura (caso clínico 1) 


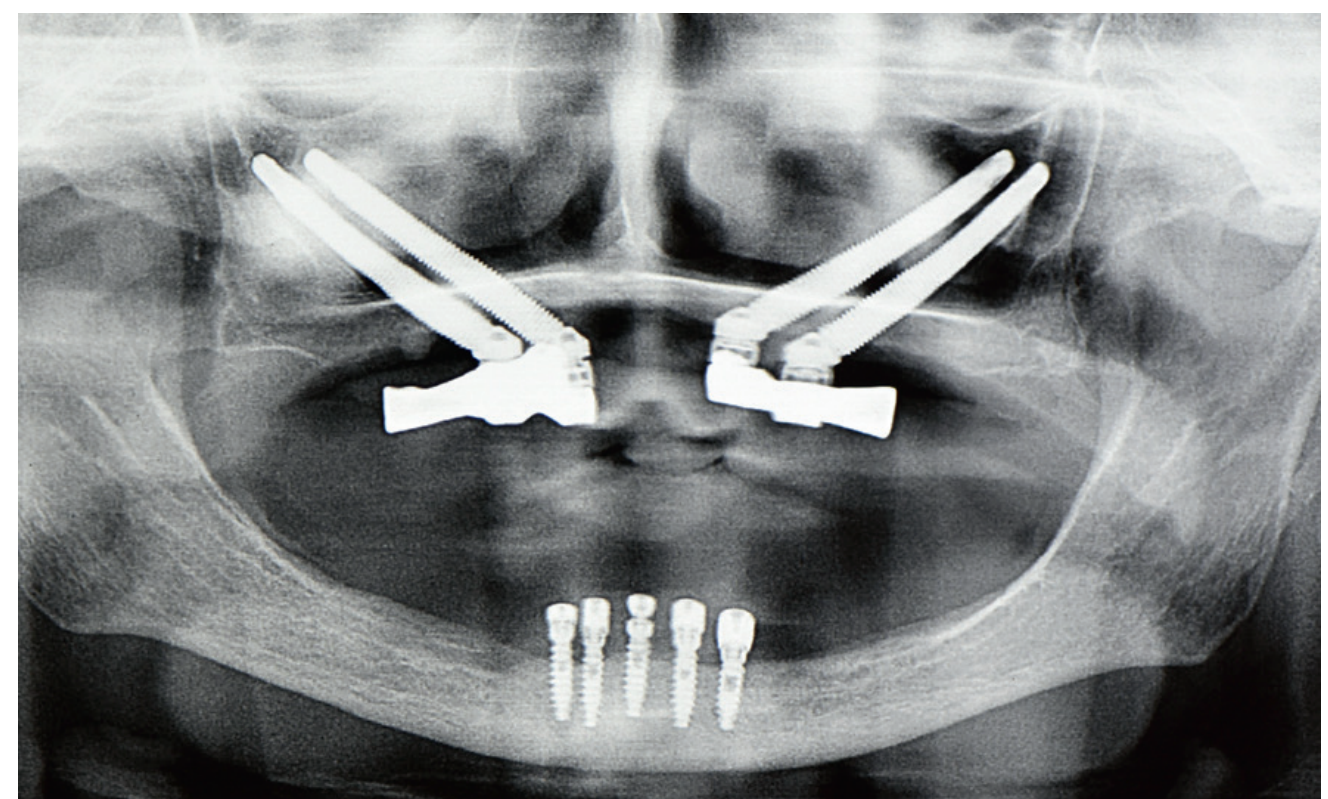

Figura 6. Radiografía panorámica 12 meses posterior a la instalación de barras protéticas (caso clínico 1)

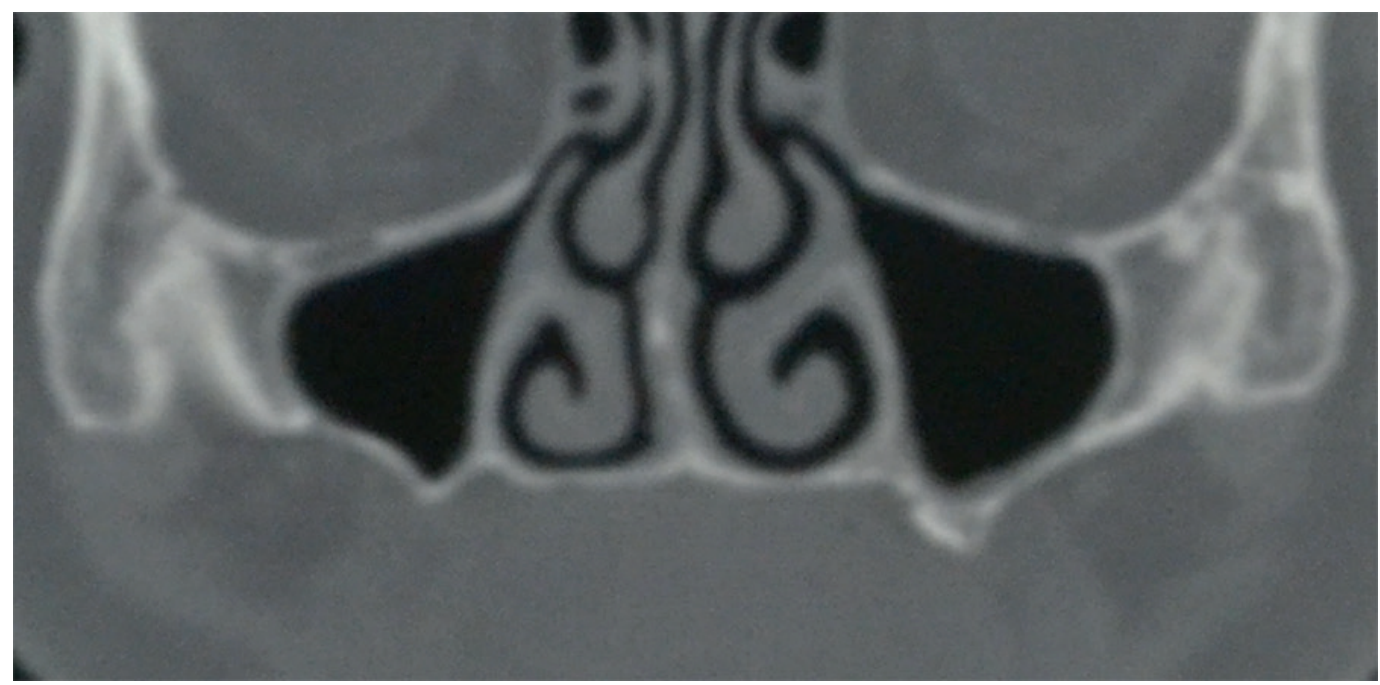

Figura 7. Corte coronal de la tomografía pre quirúrgica (caso clínico 2)

Neodent, Brasil). Se reposicionó el colgajo y se realizó la síntesis de la herida con hilo de seda. A la paciente se le administraron endovenosamente antibióticos, corticoides y analgésicos; y posteriormente, vía oral, antibióticos y analgésicos. En el post operatorio inmediato la paciente refirió dolor en la región peri orbitaria derecha. Se realizó la radiografía panorámica de control (Figura 8), y se constató que el ápice de uno de los implantes del sector, tenía una ubicación próxima a la cortical ósea del piso de orbita derecho. Se decidió con anestesia local realizar una vuelta atrás de ese implante al día siguiente a la cirugía. El dolor cesó inmediatamente a la realización de este procedimiento. No se observaron otras complicaciones en el post operatorio. A los 3 meses se realizó el destape de los implantes cigomáticos, y se realizaron los pasos protéticos. Se decidió la realización de una prótesis implanto soportada y retenida (prótesis híbrida de mediana complejidad), por preferencia de la paciente. Se rehabilitó el maxilar inferior con una prótesis parcial removible (Figura 9 y 10). Después de la carga oclusal, el paciente informó una mejoría en función y estética. Transcurridos 12 meses de la carga protética, a través de controles trimestrales, no se han observado complicaciones a nivel implantario, ni protético.

\section{Discusión}

En una revisión sistemática de implantes cigomáticos, donde se evaluaron 4556 implantes, se estableció una alta tasa de sobrevida de 95,21\%, con el mayor seguimiento en 12 años. El mayor número de fracasos se registra en los primeros 6 meses de colocados ${ }^{12}$. En un estudio clínico controlado aleatorizado donde se compararon estos implantes con implantes convencionales colocados en regeneraciones óseas, se concluyó que los implantes cigomáticos presentan mayor tasa de éxito y menores complicaciones protéticas, acortando los tiempos de tratamiento ${ }^{13}$. En los casos presentados no se ha observado fracasos a 12 y 18 meses de cargados los implantes. La oseintegración en ambos extremos 


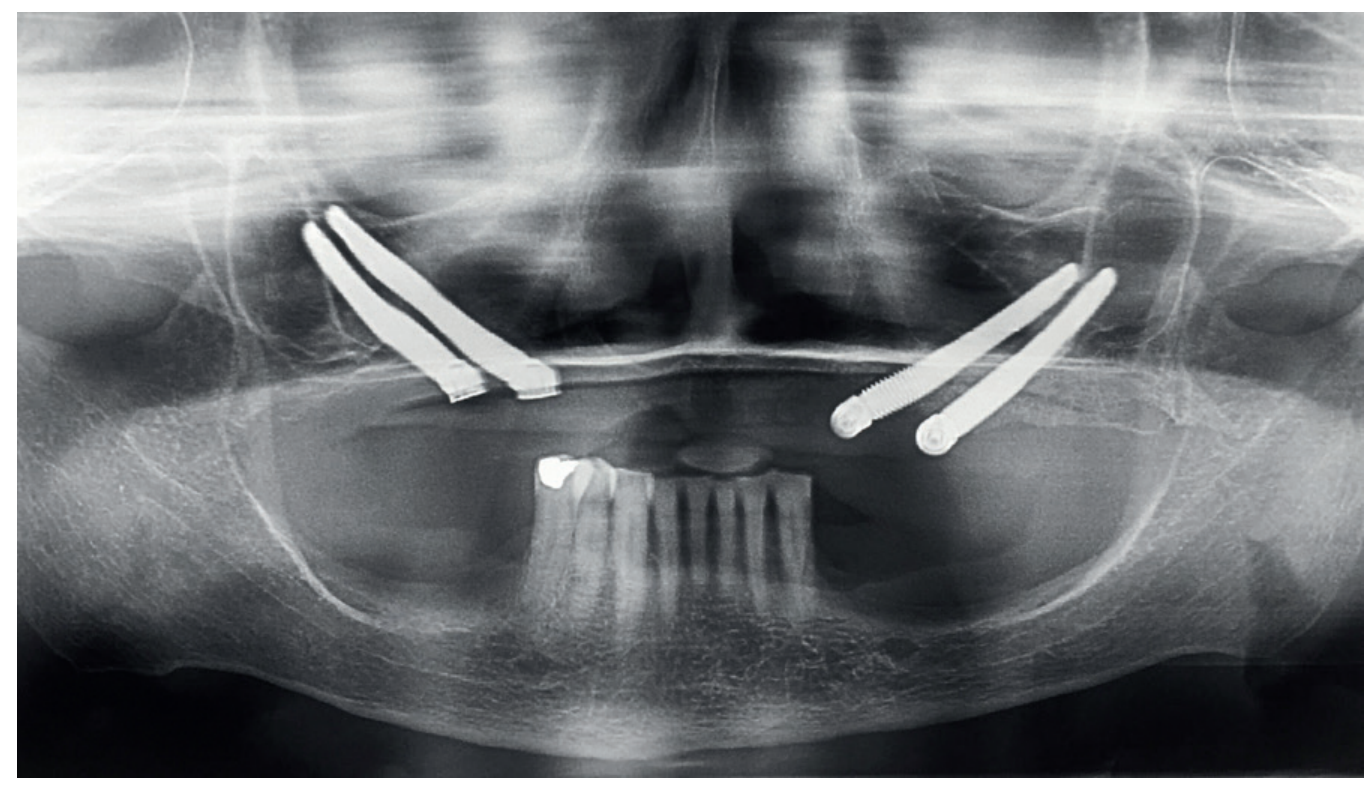

Figura 8. Radiografía panorámica post quirúrgica (caso clínico 2)

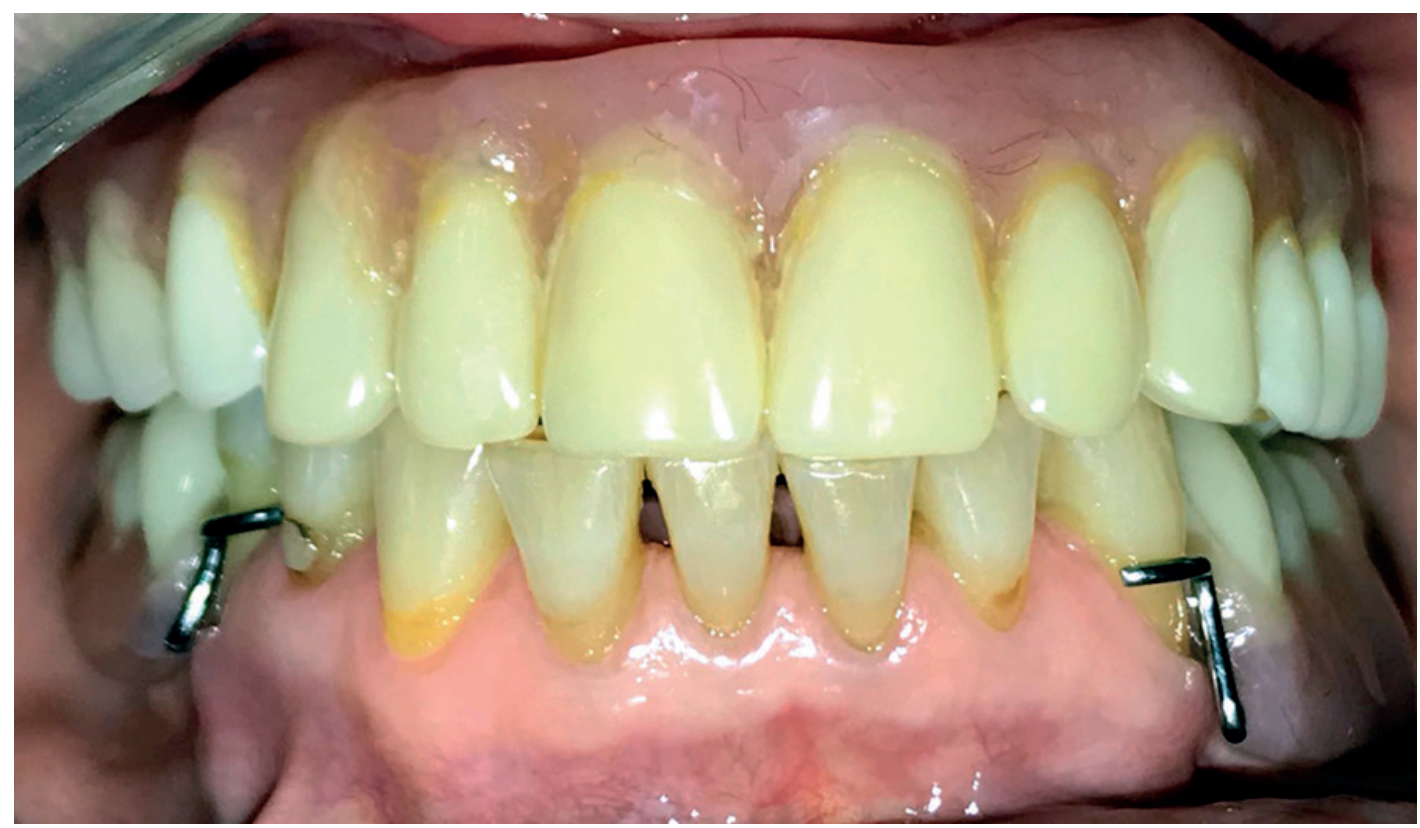

Figura 9. Rehabilitación final del maxilar superior a través de prótesis hibrida (caso clínico 2)

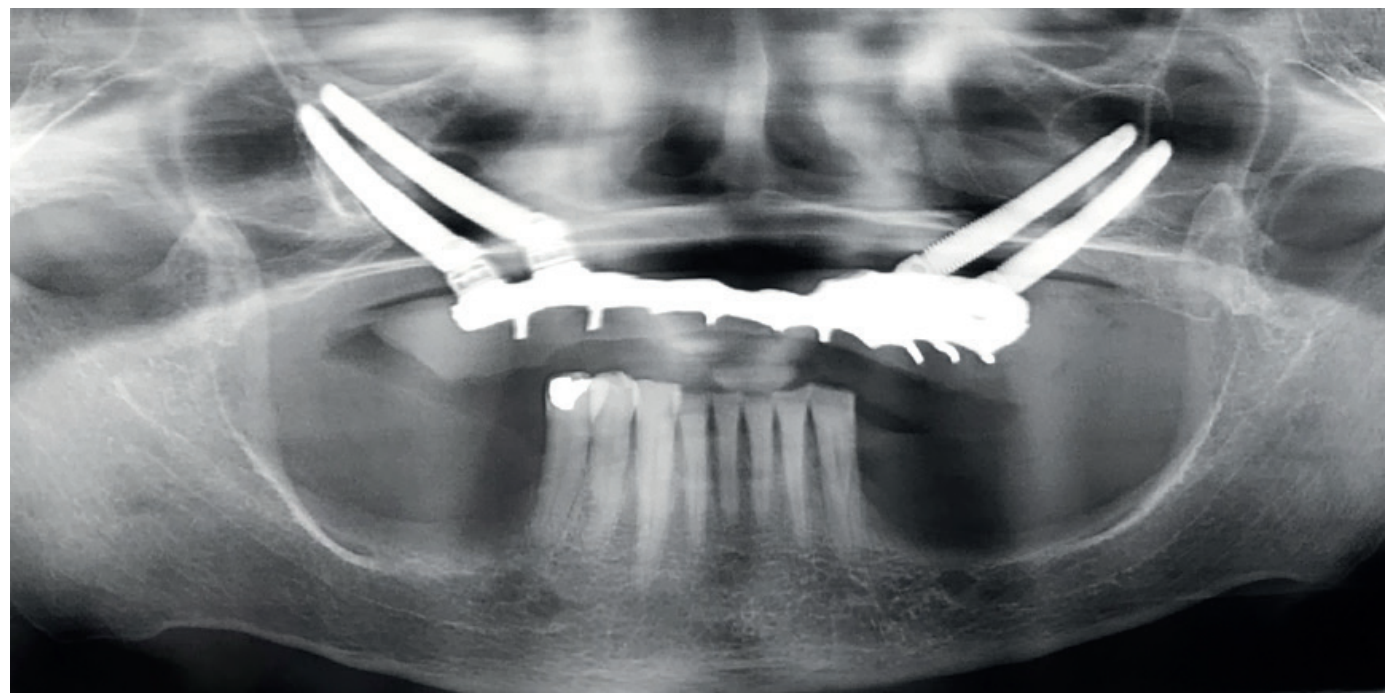

Figura 10. Radiografía panorámica 12 meses posterior a la instalación de la prótesis hibrida (caso clínico 2) 
del implante, parecerían desempeñar un rol favorable para esta sobrevida observada.

El procedimiento convencional, definido por Branemark en 1998, consistió en la colocación de un implante de 35-55 mm de longitud anclado en el hueso malar siguiendo una trayectoria intra-sinusal. Desde esta descripción, muchos autores han variado ligeramente la técnica: siguiendo el contorno del hueso malar evitando de fenestración del seno maxilar, colocándolo a vestibular del seno maxilar; combinado con implantes anteriores; reduciendo los tiempos de carga; o modificando la carga protética ${ }^{8,14-16}$. Una revisión sistemática de carga inmediata de estos implantes, concluye que es una técnica recomendable ferulizando ambos implantes ${ }^{17}$; aunque técnicamente es más complejo. La alternativa más reciente de colocar los implantes hacia vestibular tiene el objetivo de reducir la posibilidad de sinusitis post quirúrgica; aun con poca casuística, es una alternativa a considerar ${ }^{17}$. En cuanto a la combinación de implantes anteriores con implantes cigomáticos, es una alternativa con similares tasas de éxito, aunque agrega la dificultad que frecuentemente los implantes en la pre-maxila requieren regeneración ósea simultánea ${ }^{18}$. En los casos presentados, al evaluar el correcto estado y permeabilidad del seno maxilar, se decidió la colocación a través de la técnica convencional, ya que permite una muy buena visualización del sitio quirúrgico, y evita la necesidad de realizar una regeneración ósea.

Se ha documentado la colocación reversa de implantes cigomáticos. Esta técnica consiste en la colocación extra-oral de implantes diseñados para tal fin, ingresando por el malar, minimizando los riesgos de perforación del piso de la órbita ${ }^{19}$. Hoy en día, es todavía una técnica muy poco reportada para recomendar su uso.

Para la correcta colocación de los implantes cigomáticos generalmente es necesario levantar colgajo amplios para visualizar el sitio quirúrgico, y para evitar traumas en los tejidos a lo largo de la trayectoria del implante. Si bien este artículo presenta la colocación de los mismos de forma tradicional, otra alternativa es la realización de la cirugía guiada por software ${ }^{20}$. Probablemente en los años venideros, al irse perfeccionando esta tecnología, la realización de cirugías guiadas a través de guías quirúrgicas diseñadas en programas computacionales, se convierta en el tratamiento de primera elección.

La colocación de implantes cigomáticos puede presentar complicaciones, siendo la más habitual la sinusitis $(2,4 \%)$. Además, infrecuentemente se puede presentar dolor persistente, comunicación bucosinusal, infección de tejidos blandos, alteraciones sensitivas, hematoma periorbitario y sangrado nasal temporario ${ }^{12}$. En el segundo caso presentado, la paciente presentó un dolor persistente en la órbita derecha por la proximidad de un implante a la misma. Al ser girado en reversa una vuelta este dolor cesó, y el post operatorio cursó sin otras complicaciones. No se observaron complicaciones de infecciones, ni inflamatorias, ni hemorrágicas, ni sensitivas.
Desde el punto de vista protético, se han utilizado con éxito varios sistemas de fijación con sobredentaduras, que incluyen coronas telescópicas, barras, localizadores, ball atachs e imanes ${ }^{21}$. En uno de los casos se ha optado por la confección de una prótesis sobredentadura, que conlleva la ventaja de facilitar la higiene; y el otro caso se rehabilitó con una prótesis híbrida, que presenta una mayor comodidad al ser fija para el paciente. El sistema de implantes cigomáticos utilizado en las pacientes en el presente estudio, impide la colocación de una mesoestructura sin interposición de aditamentos transmucosos. Estos aditamentos, generan una complicación de espacio y emergencia en la prótesis definitiva, generando brazos de palanca menos favorables. Al realizar implantes cigomáticos, debe evaluarse no solo las características del diseño de los mismos, sino también, las alternativas de rehabilitación protética que presenta el sistema seleccionado, evaluando las alternativas que ofrece el mercado.

En conclusión, teniendo como base el éxito de los casos en el acompańamiento de 12 y 18 meses, los implantes cigomáticos son una alternativa válida para la rehabilitación implanto-protética en pacientes que presentan una atrofia severa del maxilar superior.

\section{Referencias bibliográficas}

1. Alayo Silva I, Grados Pomarino S. Rehabilitación implanto-soportada en un paciente edéntulo con maxilares atróficos. Reporte de caso. Odontol Sanmarquina. 2017;20(1):31-4. DOI: 10.15381/os.v20i1.13536.

2. Nyström E, Ahlqvist J, Gunne J, Kahnberg KE. 10-year follow-up of onlay bone grafts and implants in severely resorbed maxillae. Int J Oral Maxillofac Surg. 2004 Apr;33(3):258-62.

3. Sorní M, Guarinós J, García O, Peñarrocha M. Implant rehabilitation of the atrophic upper jaw: a review of the literature since 1999. Med Oral Patol Oral Cir Bucal. 2005 Apr 1;10 Suppl 1:E45-56.

4. Esposito M, Barausse C, Pistilli R, Piattelli M, Di Simone $S$, Ippolito DR, et al. Posterior atrophic jaws rehabilitated with prostheses supported by $5 \times 5 \mathrm{~mm}$ implants with a nanostructured calcium-incorporated titanium surface or by longer implants in augmented bone. Fiveyear results from a randomised controlled trial. Int J Oral Implantol (New Malden). 2019;12(1):39-54.

5. Peñarrocha $\mathrm{M}$, Carrillo $\mathrm{C}$, Boronat $\mathrm{A}$, Peñarrocha $\mathrm{M}$. Maximum use of the anterior maxillary buttress in severe maxillary atrophy with tilted, palatally positioned implants: a preliminary study. Int J Oral Maxillofac Implants. 2010;25(4):813-20.

6. Aparicio C, Branemark PI, MD, Keller EE, Olive J. Reconstruction of the Premaxilla With Autogenous lliac Bone in Combination With Osseointegrated Implants. Int J Oral Maxillofac Implants. 1993;8(1):61-7.

7. Weischer T, Schettler D, Mohr C. Titanium implants in the zygoma as retaining elements after hemimaxillectomy. Int J Oral Maxillofac Implants. 1997 MarApr;12(2):211-4. 
8. Chrcanovic BR, Pedrosa AR, Neto Custódio AL. Zygomatic implants: a critical review of the surgical techniques. Oral Maxillofac Surg. 2013 Mar;17(1):1-9. DOI: $10.1007 / s 10006-012-0316-y$.

9. Nkenke E, Hahn M, Lell M, et al. Anatomic site evaluation of the zygomatic bone for dental implant placement. Clin Oral Implants Res 2003;14(1):72-9.

10. Duarte LR, Filho HN, Francischone CE, Peredo LG, Branemark PI. The establishment of a protocol for the total rehabilitation of atrophic maxillae employing four zygomatic fixtures in an immediate loading system-A 30 -month clinical and radiographic follow-up. Clin Implant Dent Relat Res 2007;9(4):186-196.

11. Bothur S, Kindberg H, Lindqvist J. The positions of implant heads in relation to the fixed dental prosthesis: A comparison of multiple zygomatic implants with standard implants for the reconstruction of the atrophic maxilla. Int J Oral Maxillofac Implants 2012;27:664-670.

12. Chrcanovic BR, Albrektsson T, Wennerberg A. Survival and Complications of Zygomatic Implants: An Updated Systematic Review. J Oral Maxillofac Surg. 2016 Oct;74(10):1949-64. DOI: 10.1016/j. joms.2016.06.166.

13. Esposito M, Davó R, Marti-Pages C, Ferrer-Fuertes A, Barausse C, Pistilli R, Ippolito DR, Felice P. Immediately loaded zygomatic implants vs conventional dental implants in augmented atrophic maxillae: 4 months postloading results from a multicentre randomised controlled trial. Eur J Oral Implantol. 2018;11(1):11-28.

14. Balan I, DI Girolamo M, Lauritano D, Carinci F. Treatment of severe atrophic maxilla with zygomatic implants: a case series. Oral Implantol (Rome). 2017 Nov 30;10(3):317-324. DOI: 10.11138/orl/2017.10.3.317.
15. Galán-Gil S, Peñarrocha-Diago M, Balaguer-Martínez J, Marti-Bowen E . Rehabilitation of severely resorbed maxillae with zygomatic implants: an update. Med Oral Patol Oral Cir Bucal 2007;12(3):E216-20.

16. Petrungaro PS, Kurtzman GM, Gonzales S, Villegas C. Zygomatic Implants for the management of severe alveolar atrophy in the partial or completely edentulous maxilla. Compend Contin Educ Dent. 2018 Oct;39(9):636645.

17. Tuminelli FJ, Walter LR, Neugarten J, Bedrossian E. Immediate loading of zygomatic implants: A systematic review of implant survival, prosthesis survival and potential complications. Eur J Oral Implantol. 2017;10 Suppl $1: 79-87$.

18. Aboul-Hosn Centenero S, Lázaro A, Giralt-Hernando M, Hernández-Alfaro F. Zygoma Quad Compared With 2 Zygomatic Implants: A Systematic Review and Meta-analysis. Implant Dent. 2018 Jan 29. DOI: 10.1097/ ID.0000000000000726.

19. Dawood A, Collier J, Darwood A, Tanner S. The reverse zygomatic implant: a new implant for maxillofacial reconstruction. Int J Oral Maxillofac Implants. 2015 NovDec;30(6):1405-8. DOI: 10.11607/jomi.4556.

20. Chow J. A novel device for template-guided surgery of the zygomatic implants. Int J Oral Maxillofac Surg. 2016 Oct;45(10):1253-5. DOI: 10.1016/j.ijom.2016.06.007.

21. Savabi O, Nejatidanesh F, Yordshahian F. Retention of implant-supported overdenture with bar/clip and stud attachment designs. J Oral Implantol. 2013;39(2):1407. DOI: 10.1563/AAID-JOI-D-11-00016. 